Int. J. Odontostomat.,

10(3):507-512, 2016

\title{
Quiste Epidermoide Gigante en Reloj de Arena del Piso de la Cavidad Oral
}

\author{
Giant Hourglass Shaped Epidermoid Cyst in the Floor of Mouth
}

\author{
Loreto Canto C.; Fernanda Pintor W."; María de Los Ángeles Fernández T.*; \\ Matteo De La Fuente $A^{\star * * *}$. \& Carlos Bahamondes A.*
}

CANTO, C. L.; PINTOR, W. F.; FERNÁNDEZ, T. M. Á.; DE LA FUENTE, A. M. \& BAHAMONDES, A. C. Quiste epidermoide gigante en reloj de arena de la cavidad oral. Int. J. Odontostomat., 10(3):507-512, 2016.

RESUMEN : El quiste epidermoide es un quiste de tejido blando de origen ectodérmico que se encuentra con poca frecuencia en la cavidad oral. Clínicamente se caracteriza por ser una lesión asintomática, de larga evolución, recubierta con mucosa normal, y usualmente se diagnostica cuando el aumento de volumen genera molestias en el paciente. El tratamiento varía de acuerdo a su tamaño y localización anatómica. Presentamos el caso de una paciente de 29 años de edad con un quiste epidermoide de gran tamaño, en reloj de arena, del piso de boca. Se describen las características específicas histológicas y clínicas, y se discute la conducta terapéutica seguida.

PALABRAS CLAVE: quiste epidermoide, quiste dermoide, lesión benigna.

\section{INTRODUCCIÓN}

El quiste epidermoide (QE) es una malformación quística del desarrollo, que se presenta de manera poco frecuente, con una incidencia en cabeza y cuello que va del $1,6 \%$ al $6,9 \%$, y representa el 0,01 $\%$ de todos los quistes de la cavidad oral (Mohta \& Sharma, 2006).

Existen múltiples teorías sobre la etiología de esta patología, la primera atribuye el origen a un crecimiento de tejido ectodérmico atrapado del primer y segundo arco branquial. La segunda, sugiere eventos quirúrgicos o accidentales que generan una implantación traumática de células epiteliales en tejidos más profundos, y una tercera teoría considera la posibilidad de que sea una variante del quiste del conducto tirogloso (De Mello et al., 1987; King et al., 1994 \& De Ponte, 2002.).

Clínicamente, el QE se presenta como un aumento de volumen de crecimiento lento, progresivo, asintomático y de consistencia pastosa, generalmente entre la $1^{\circ}$ y $3^{\circ}$ décadas de vida.
Su ubicación más frecuente es el piso de la cavidad oral, pudiendo tener distintas presentaciones dependiendo de su localización con respecto al músculo milohioideo. Cuando el crecimiento es por sobre este músculo, produce glosoptosis resultando potencialmente en disfagia, disfonía y disnea. Cuando el crecimiento es por debajo del músculo, se presenta como un aumento de volumen submental (Neville et al., 2009; Chaudhry et al., 2013; Mesolella et al., 2013; Gaddikeri et al., 2014 \& Tandon \& Gupta, 2014).

El mecanismo de crecimiento de esta patología es desconocido, algunos autores plantean que existiría una asociación entre el cambio hormonal post puberal y el acelerado crecimiento de este quiste.

Histológicamente, se caracteriza por presentar espacios quísticos tapizados por epitelio escamoso simple, pero sin apéndices dérmicos en la pared quística, aspecto que lo diferencia del quiste dermoide (Neville et al.).

* Cirujano Buco Maxilofacial, Universidad de Chile.

* Cirujano Buco Maxilofacial, Hospital Clínico Universidad de Chile.

*** Cirujano Buco Maxilofacial, Hospital San Juan de Dios, Chile.

${ }^{* * * *}$ Cirujano Dentista. Departamento de Cirugía y Traumatología Buco Maxilofacial, Universidad de Chile.

**o*** Residente Cirugía Buco Maxilofacial, Universidad Mayor. 
Con respecto al tratamiento, la enucleación completa de la lesión es lo indicado y el abordaje quirúrgico dependerá de su tamaño y ubicación, siendo vía oral si la lesión se encuentra sobre el músculo milohioideo, y cervical si la ubicación es por debajo de éste (Gómez-Carrillo et al., 2011).

A continuación se presenta un caso clínico de un QE de gran tamaño en el piso de la cavidad oral y se discute con respecto a la terapéutica utilizada.

\section{CASO CLÍNICO}

Paciente de género femenino de 29 años de edad, fue derivada desde un centro de atención primaria de salud, al hospital San Juan de Dios (San-

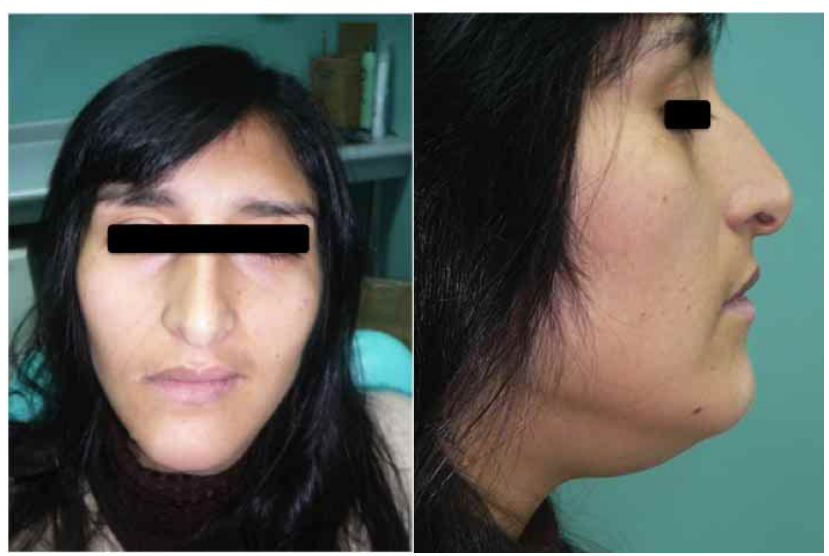

Fig. 1. Fotografía facial de la paciente en la cual puede observarse un aumento de volumen submental y submandibular izquierdo. La piel que cubre la lesión es normal.

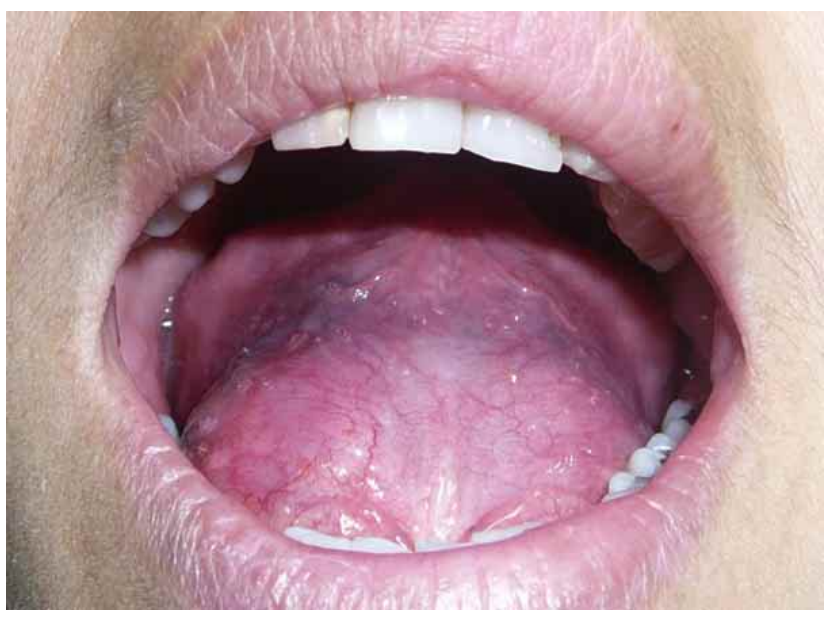

Fig. 2. Fotografía oral en la cual se aprecia un gran aumento de volumen del piso de la boca que desplaza a la lengua en sentido posterosuperior. La mucosa se encuentra indemne.

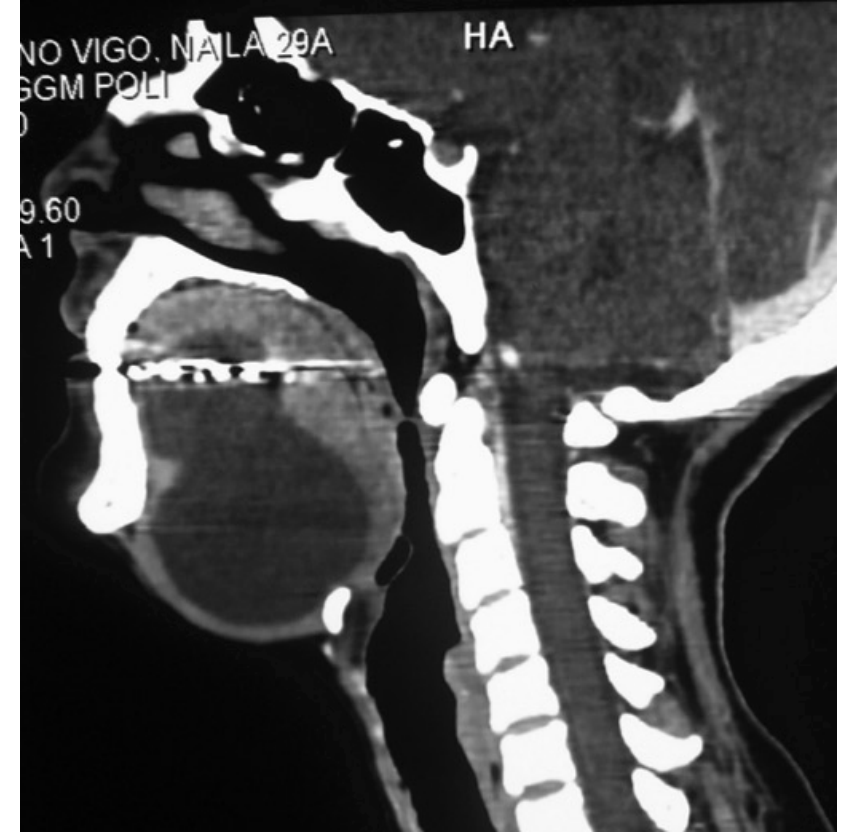

Fig. 3. TC, corte sagital. Se observa extensa lesión quística que compromete la region sublingual, atraviesa el músculo milohioideo comprometiendo además el espacio submental, desplazando tejidos y disminuyendo la vía aérea.

tiago, Chile) para la evaluación de un aumento de volumen en la zona sublingual y submental, de 2 años de evolución. La paciente relató que inicialmente la lesión comprometía sólo el piso de la cavidad oral, y que, progresivamente fue aumentando de tamaño. Relataba además que aumentaba de tamaño cuando se acercaban las comidas.

La paciente no presentaba antecedentes mórbidos ni quirúrgicos relevantes sólo un embarazo el último año, tiempo durante el cual se percató de un crecimiento constante y significativo de la lesión lo cual le provoco alteraciones de su fonación, situación que la llevo a consultar.

Al examen facial se apreció un aumento de volumen que comprometía la región submental y submandibular del lado izquierdo, de consistencia blanda, depresible y levemente doloroso a la palpación, sin compromiso de la piel en relación a la lesión (Fig.1).

Al examen intraoral la paciente presentaba una extensa lesión que comprometía ambos compartimientos sublinguales y generaba un desplazamiento posterosuperior lingual, la mucosa que lo recubría estaba indemne. Era de consistencia blanda, depresible y sensible a la palpación (Fig. 2). 


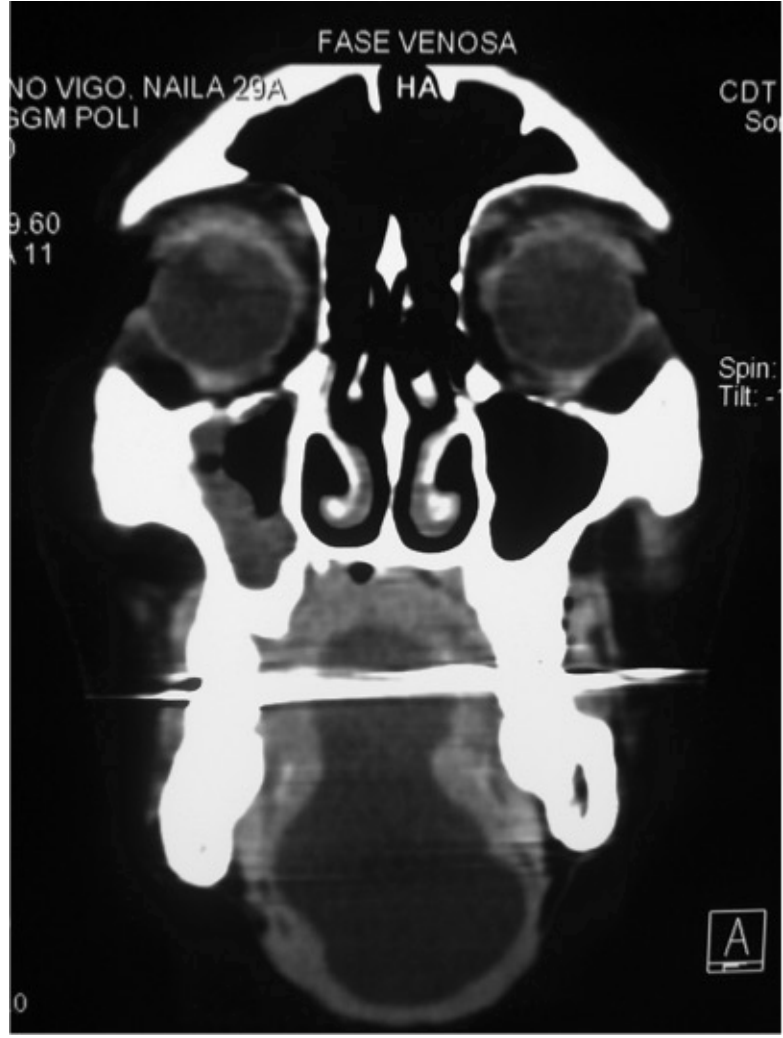

Fig. 4. TC, corte coronal. Se observa el gran compromiso de la zona submentoniana y del espacio submandibular del lado izquierdo.

En el corte sagital de la tomografía computada (TC), se pudo observar una gran lesión hipodensa de aspecto quístico en el espacio sublingual que atravesabacaudalmente el músculo milohioídeo, generando un gran aumento de volumen y desplazamiento de tejidos de la región submental. La lesión generaba un desplazamiento posterosuperior lingual que disminuía el lumen anteroposterior de la hipofaringe (Fig. 3). En el corte coronal pudo apreciarse especialmente el compromiso de la zona submental y la constricción que provocaba en el músculo milohioideo al ser atravesado por la lesión, generando un aspecto característico de "reloj de arena". Además se observó un leve desplazamiento lateral de la lesión hacia el lado izquierdo, denotando compromiso del espacio submandibular (Fig. 4).

Según la historia clínica, examen físico e imagenológico se plantea como hipótesis diagnóstica una ránula en reloj de arena. Se plantea, por lo tanto, como tratamiento la enucleación completa de la lesión a través de un acceso intraoral y la sublinguectomía correspondiente.
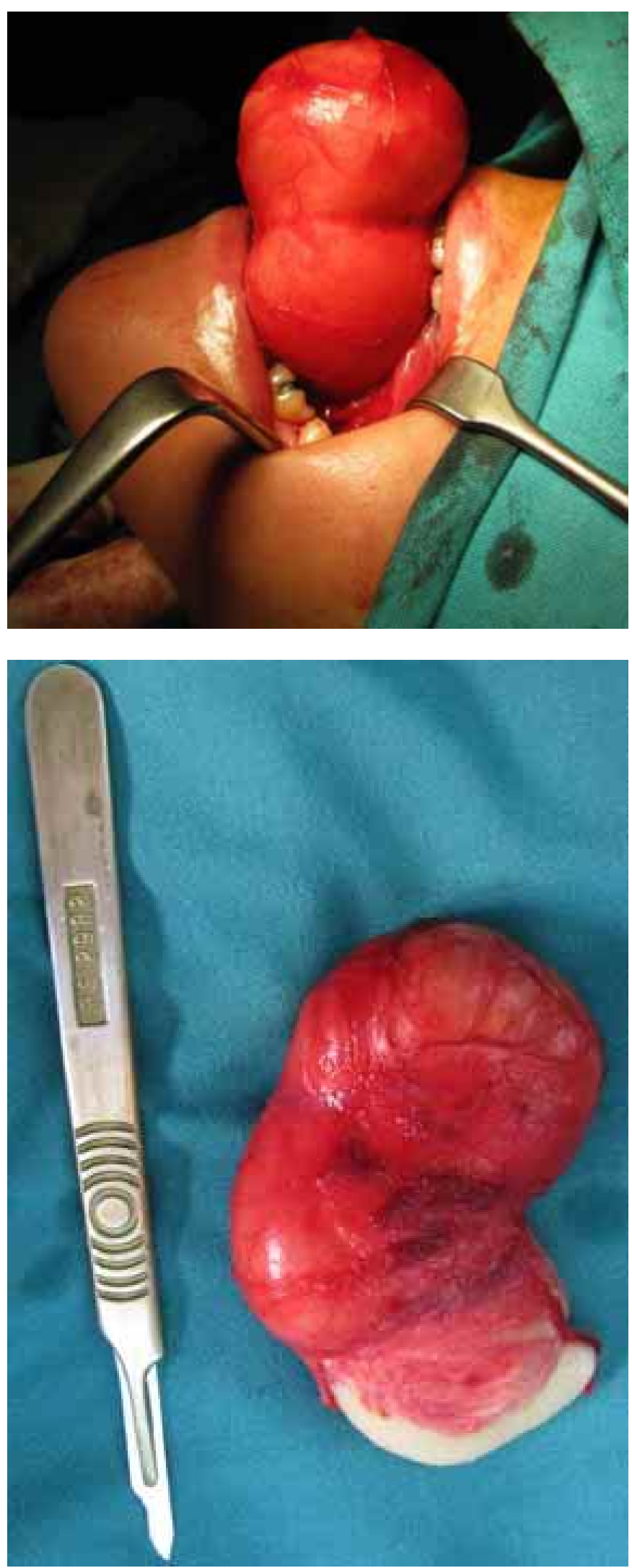

Figs. 5 y 6 . Enucleación quirúrgica de la lesión vía intraoral. 
Bajo anestesia general, se realizó una incisión en la mucosa del piso de cavidad oral, a nivel del límite entre la base de la lengua y el borde superior de la lesión. Comienza la disección de la lesión encontrando con facilidad el plano de clivaje, situación no característica de una ránula. Se realiza su remoción completa vía intraoral disecando a través del músculo milohioideo hasta el espacio submental para eliminarla completamente. La lesión enucleada es identificada en el intraoperatorio como un quiste epidermoide de $6,7 \times 5 \times 3$ centímetros de tamaño (Figs. 5 y 6 ) por lo que se acondiciona la cavidad, se identifica la indemnidad de elementos nobles, se deja la glándula sublingual en su lugar y se realiza el cierre. El periodo postoperatorio mediato no presentó complicaciones.

En el estudio histopatológico se observó una cavidad quística recubierta por epitelio plano
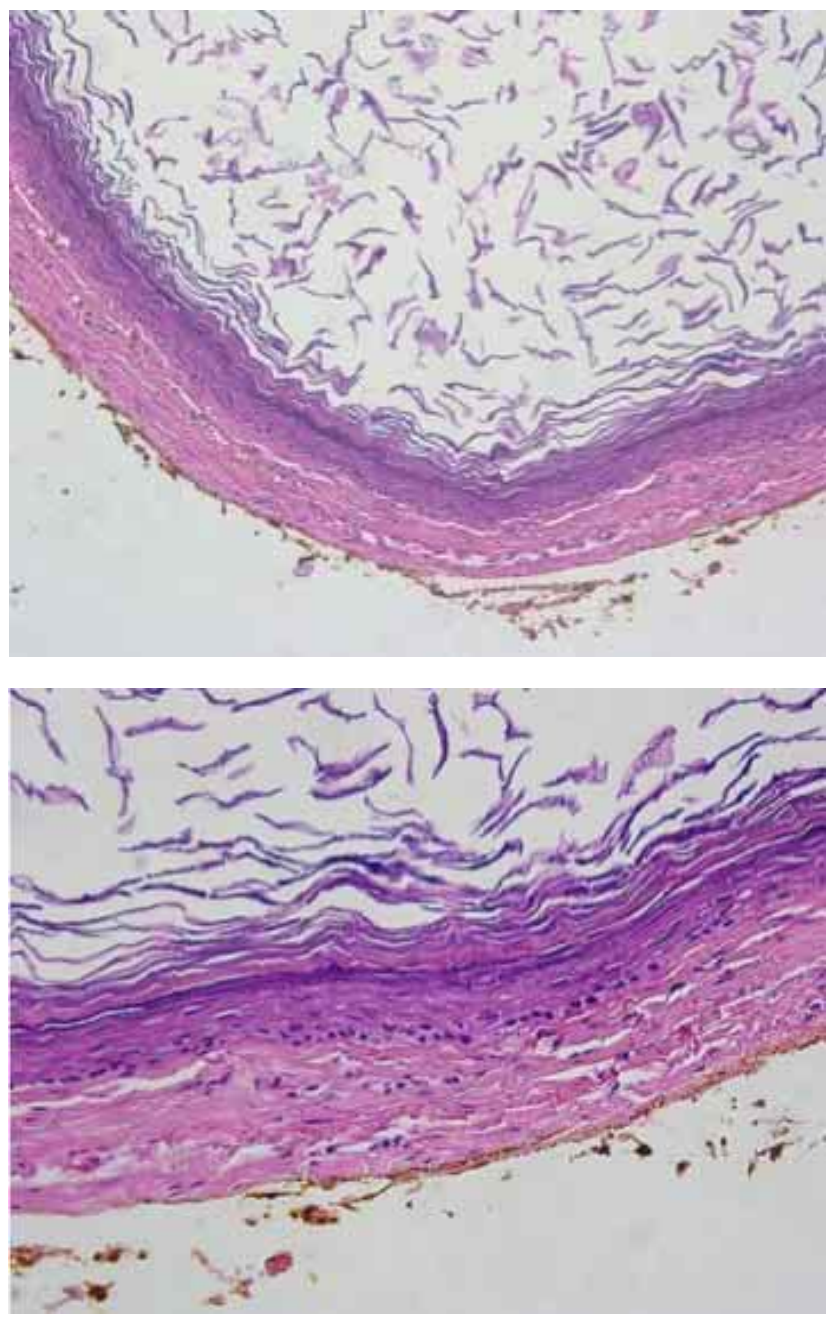

Figs. 7 y 8 . Imagen de (100x H-E) (400x H-E) microfotografía que muestra un epitelio plano pluriestratificado ortoqueratinizado y una capsula de tejido conectivo fibroso. pluriestratificado, con tejido conectivo fibroso subyacente presentando al interior cantidades variables de queratina (Figs. 7 y 8 ). El diagnóstico definitivo corresponde al de quiste epidermoide.

La paciente se mantuvo con controles periódicos en el post operatorio inmediato y tardío y después 4 años de operada, se encontrabra en buenas condiciones.

\section{DISCUSIÓN}

El quiste epidermoide (QE) es una lesión congénita de tejido blando, circunscrita, de lento crecimiento y asintomática. Generalmente pasa desapercibida por su pequeño tamaño por lo que se diagnóstica entre la segunda y tercera década de vida, ya que los pacientes consultan cuando la lesión ha alcanzado un gran volumen y produce alteraciones estéticas o funcionales como lo son; la deglución, fonoarticulación y respiración en casos que se ubique en el piso de la cavidad oral (Chaudhry et al. \& Gulati et al., 2015).

Cuando hablamos de quiste epidermoide, es necesario aclarar que esta lesión es una subdivisión histológica del quiste dermoide, el cual se subdivide en tres lesiones; quiste dermoide verdadero, quiste epidermoide y teratoma. La variedad epidermoide se compone solamente de elementos de la epidermis y carece de anexos de la piel como folículos pilosos o glándulas sebáceas. Éste contiene material turbio compuesto principalmente de células descamadas (Gulati et al).

Habitualmente el QE es una lesión cutánea ubicada en la cara, cuero cabelludo, cuello y torso (Dutta et al., 2013). Es raro encontrar esta lesión en la cavidad oral, ya que dentro de todas las ubicaciones en el cuerpo, la cavidad oral ocupa sólo un 1,6 \% de los sitios afectados por este quiste (Chaundry et al.). Debido a la baja incidencia en la cavidad oral del QE, además de la historia de la lesión y su presentación clínica particularmente como reloj de arena, éste no fue considerado como hipótesis diagnóstica principal, por el contrario se determinó que se trataba de una ránula en reloj de arena, que es una lesión exclusiva del piso de cavidad oral.

El piso de cavidad oral y el espacio submandibular pueden ser afectados por numerosas patologías, que se pueden clasificar ampliamente en inflamatorias, obstructivas o neoplásicas. Dentro del 
diagnóstico diferencial de patologías ubicadas en ésta zona se encuentra el quiste dermoide, lipoma, quiste del conducto tirogloso, ránula, malformaciones linfáticas, infecciones de glándulas submaxilar o sublingual entre otros (Verma et al., 2012 \& Baliga et al., 2014).

En este caso la paciente relató haber percibido un incremento de tamaño progresivo de la lesión, sin percatarse que el mayor aumento de tamaño pudo haberse producido asociado a su último embarazo. Mesolella et al. reportaron el caso de una paciente de 27 años embarazada con 26 semanas de gestación que presentaba un quiste dermoide. La paciente relató haber padecido de esta masa desde los 14 años y que durante su embarazo comenzó a crecer rápidamente. A pesar de que se desconoce el mecanismo exacto por el cual estos quistes crecen durante el embarazo, realizaron estudios inmunohistoquímicos de la muestra obtenida y observó que el quiste expresó un elevado número de receptores para estrógeno y progesterona, hormonas que se presentan elevadas durante el embarazo, por lo que planteó la hipótesis de que estas hormonas podrían actuar como factores de crecimiento del quiste.

Como exámenes complementarios para el diagnóstico y estudio de la lesión se recomienda el uso de resonancia magnética (RNM) y tomografía computarizada (TC), ya que permiten observar la extensión total de la lesión y la relación del quiste con estructuras anatómicas vecinas. También se describe el uso de punción aspirativa con aguja fina como método de diagnóstico seguro y de bajo costo, lo cual podría haber sido recomendable en este caso para confirmar o descartar la hipótesis diagnóstica planteada, pero debe ser complementada con exámenes imagenológicos como TC y RNM (Verma et al. \& Tandon et al).

En este caso el abordaje escogido fue vía intraoral debido a nuestra hipótesis diagnóstica, ya que el tratamiento contemplaba realizar la exéresis de la lesión, pero principalmente la glándula sublingual que la originaba. Al encontrar un buen plano de clivaje durante el procedimiento e identificar características macroscópicas diferentes a las de una ránula, se continuó con la enucleación de la lesión in toto, la cual luego se identificó como quiste epidermoide debido al contenido encontrado. El abordaje intraoral permite la eliminación completa de la lesión y presenta menor tasa de complicaciones post operatorias en comparación a un abordaje submental; permitiendo una restitución tisular integral y evitando la cicatriz visible de un abordaje por piel (Gómez-Carrillo et al.).

Existe una variedad de patologías que afectan el piso de cavidad oral, siendo el quiste epidermoide una de las menos frecuentes de ellas, sin embargo, igual debe ser parte de nuestro diagnóstico diferencial. Es importante realizar una historia clínica acabada para determinar la etiología y la evolución del proceso patológico que afecta al paciente y así mismo es fundamental realizar un análisis imagenológico minucioso para la planificación del acto quirúrgico y determinar la eventual necesidad de otros elementos diagnósticos coadyudantes.

CANTO, C. L.; PINTOR, W. F.; FERNÁNDEZ, T. M. Á.; DE LA FUENTE, A. M. \& BAHAMONDES, A. C. Giant hourglass shaped epidermoid cyst in the floor of mouth. Int. J. Odontostomat., 10(3):507-512, 2016.

ABSTRACT: The epidermoid cyst is a soft tissue cyst of ectodermal origin found infrequently in the oral cavity. Clinically, it is characterized by an asymptomatic lesion, of long evolution, covered with normal mucosa, and is usually diagnosed when the volume increases and generates patient discomfort. Treatment varies according to its size and anatomical location. We report the case of a 29 year old patient with a large, hourglass shaped, epidermoid cyst in the floor of the mouth. Specific histological and clinical characteristics are described and the therapeutic behavior followed is discussed.

KEY WORDS: epidermoid cyst, dermoid cyst, benign lesion.

\section{REFERENCIAS BIBLIOGRÁFICAS}

Baliga, M.; Shenoy, N.; Poojary, D.; Mohan, R. \& Naik, R. Epidemroid cyst of the floor of the mouth. Natl. J. Maxillofac. Surg., 5:79-83, 2014.
Chaudhry, S.; Hannan, A. \& Ikram, U. Adult woman with intraoral epidermoid cyst. J. Ayub. Med. Coll. Abbottabad, 25:213-14, 2013. 
CANTO, C. L.; PINTOR, W. F.; FERNÁNDEZ, T. M. Á.; DE LA FUENTE, A. M. \& BAHAMONDES, A. C. Quiste epidermoide gigante en reloj de arena de la cavidad oral. Int. J. Odontostomat., 10(3):507-512, 2016.

De Mello, D.; Lima, J. \& Liapis, H. Midline cervical cysts in children. Arch. Otolaryngol. Head and Neck Surg., 113:418-20, 1987.

De Ponte, F. S. Sublingual epidermoid cyst. J. Craniofac. Surg., 13:308-10, 2002.

Dutta, M.; Saha, J.; Biswas, G. et al. Epidemroid cysts in the head and neck: our experiences with, review of literature. Indian J. Otolaryngol. Head Neck Surg., 65:14-21, 2013.

Gaddikeri, S.; Vattoth, S.; Gaddikeri, R. et al. Congenital cystic neck masses: embryology and imagind appearances, with clinicopathological correlation. Curr. Probl. Diagn. Radiol., 43:55-67, 2014.

Gómez-Carrillo, V.; Martínez-Seijas, P.; Díaz-Galvis, L. et al. Técnica quirúrgica para abordaje intraoral de quiste dermoide cervical de gran tamaño: a propósito de un caso clínico. Rev. Esp. Cir. Oral Maxilofac., 33:124-128, 2011.

Gulati, U.; Mohanty, S.; Augustine, J, \& Gupta, S. R. Potentially fatal supramylohyoid sublingual epidermoid cyst. J. Maxillofac. Oral Surg., 14:355-9, 2015.

King, R.; Smith, B. \& Burk, J. Dermoid cyst in the floor of the mouth. Oral Surg. Oral Med. Oral Pathol., 78:567-76, 1994.

Mesolella, M.; Cantone, E.; Galli, V. \& lengo, M. Challanging management of giant sublingual dermoid cyst rapidly enlarged throughout pregnancy and influence of hormonal factors. Surgical Science, 4:216-218, 2013.

Mohta, A. \& Sharma, M. Congenital oral cysts in neonates: Report of two cases. Oral Surg Oral Med. Oral Pathol. Oral Radiol. Endod., 102:36-8, 2006.

Neville, B. W.; Damm, D. D.; Allen, C. M. \& Bouquot, J. E. Oral \& Maxillofacial Pathology. 3rd ed. St. Louis, Mo.: Saunders/?Elsevier, 2009.

Tandon, P. N. \& Gupta, D. S. Epidermoid cyst in the floor of mouth with sub mental component. J. Maxillofac. Oral Surg., 13:59-62, 2014.

Verma, S.; Kushwaha, J. K.; Sonkar, A. A.; Kumar, R. \& Gupta, R. Giant sublingual epidermoid cyst resembling plunging ranula. Natl. J. Maxillofac. Surg., 3:211-213, 2012.
Dirección para correspondencia:

Dr. Matteo De La Fuente A.

Cirujano Dentista

Departamento de Cirugía y

Traumatología Buco Maxilofacial

Universidad de Chile

Santiago

CHILE

Email: matteodelafuentea@gmail.com

Recibido: 16-07-2016

Aceptado:18-08-2016 